Address correspondence to: Ronjon Chakraverty, Transplantation Immunology Group, Cancer Institute and Institute for Immunity and Transplantation, University College London, London, United Kingdom. Phone: 44.207.472.6100; Fax: 44.207.830.2092; E-mail: r.chakraverty@ucl.ac.uk.

1. Rondina MT, Weyrich AS, Zimmerman GA. Platelets as cellular effectors of inflammation in vascular diseases. Circ Res. 2013;112(11):1506-1519.

2. Sachais BS, Higazi AA, Cines DB, Poncz M, Kowalska MA. Interactions of platelet factor 4 with the vessel wall. Semin Thromb Hemost. 2004;30(3):351-358.

3. Kowalska MA, Rauova L, Poncz M. Role of the platelet chemokine platelet factor 4 (PF4) in hemostasis and thrombosis. Thromb Res. 2010;125(4):292-296.

4. Lambert MP, Rauova L, Bailey M, Sola-Visner MC, Kowalska MA, Poncz M. Platelet factor 4 is a negative autocrine in vivo regulator of megakaryopoi- esis: clinical and therapeutic implications. Blood. 2007;110(4):1153-1160.

5 . Koenen RR, et al. Disrupting functional interactions between platelet chemokines inhibits atherosclerosis in hyperlipidemic mice. Nat Med. 2009; 15(1):97-103.

6. Dudek AZ, Nesmelova I, Mayo K, Verfaillie CM, Pitchford S, Slungaard A. Platelet factor 4 promotes adhesion of hematopoietic progenitor cells and binds IL-8: novel mechanisms for modulation of hematopoiesis. Blood. 2003;101(12):4687-4694.

7. Baldwin WM 3rd, Kuo HH, Morrell CN. Platelets: versatile modifiers of innate and adaptive immune responses to transplants. Curr Opin Organ Transplant. 2011;16(1):41-46.

8. Shi G, et al. Platelet factor 4 limits Th17 differentiation and cardiac allograft rejection. J Clin Invest. 2014;124(2):543-552.

9. Yuan X, et al. A novel role of CD4 Th17 cells in mediating cardiac allograft rejection and vasculopathy. J Exp Med. 2008;205(13):3133-3144.

10. Basu R, Hatton RD, Weaver CT. The Th17 family: flexibility follows function. Immunol Rev. 2013; 252(1):89-103.

11. Boilard E, et al. Platelets amplify inflammation in arthritis via collagen-dependent microparticle production. Science. 2010;327(5965):580-583.

12. Lasagni $L$, et al. An alternatively spliced variant of CXCR3 mediates the inhibition of endothelial cell growth induced by IP-10, Mig, and I-TAC, and acts as functional receptor for platelet factor 4. J Exp Med. 2003;197(11):1537-1549.

13. de Latour RP, et al. Th17 immune responses contribute to the pathophysiology of aplastic anemia. Blood. 2010;116(20):4175-4184.

14. Johnsen J. Pathogenesis in immune thrombocytopenia: new insights. Hematology Am Soc Hematol Educ Program. 2012;2012:306-312.

15. Serody JS, Hill GR. The IL-17 differentiation pathway and its role in transplant outcome. Biol Blood Marrow Transplant. 2012;18(1 suppl):S56-S61.

16. Akpek G, et al. Development of a prognostic model for grading chronic graft-versus-host disease. Blood. 2001;97(5):1219-1226.

\title{
Lipids rule: resetting lipid metabolism restores T cell function in systemic lupus erythematosus
}

\author{
Yoko Kidani ${ }^{1}$ and Steven J. Bensinger ${ }^{1,2,3,4}$
}

\begin{abstract}
1Department of Pathology and Laboratory Medicine, ${ }^{2}$ Department of Molecular and Medical Pharmacology, and ${ }^{3} U C L A$ Metabolomics Center, David Geffen School of Medicine, UCLA, Los Angeles California, USA. ${ }^{4}$ Jonsson Comprehensive Cancer Center, UCLA, Los Angeles California, USA.
\end{abstract}

\begin{abstract}
Systemic lupus erythematosus (SLE) is a devastating autoimmune disease characterized by chronic inflammation and systemic destruction of host organs or tissue. A key feature of SLE is T cell dysfunction characterized by hyperresponsive antigen receptor signaling. In this issue of the JCI, McDonald and colleagues provide evidence that homeostasis of a subset of lipids, the glycosphingolipids (GSLs), is severely perturbed in the membranes of $T$ cells from SLE patients. Furthermore, normalization of GSLs restored TCR signaling and ameliorated $T$ cell dysfunction. These data suggest that targeting host metabolism may be an effective means of reinforcing selftolerance and attenuating autoimmunity.
\end{abstract}

Systemic lupus erythematosus (SLE) is a systemic autoimmune disease characterized by immune hyperactivity and loss of immunologic tolerance to self-antigens. Clinical manifestations often include symptoms of chronic inflammation, such as fatigue and fevers, as well as more specific features, including skin rashes, renal disease, arthritis, cardiovascular disease, vasculopathies, coagulopathies, and CNS involvement. The etiology of SLE is not well understood and likely includes both environmental and genetic factors. However, it is clear that dysfunction of multiple facets of host immunity underlies SLE pathogen-

Conflict of interest: The authors have declared that no conflict of interest exists.

Citation for this article: $J$ Clin Invest. 2014;

124(2):482-485. doi:10.1172/JCI74141 esis, resulting in inflammatory immune cell infiltrates, autoantibody production, and deposition of pathogenic antibodies in target organs (reviewed in ref. 1).

\section{Lipid dysfunction \\ in autoimmune disease}

Lipid dysfunction is a common clinical observation in many patients with rheumatic diseases and results in a heightened risk of cardiovascular disease independent of therapy (2-4). Genetic deletion of key proteins, such as apolipoprotein E, involved in lipid homeostasis has also been shown to exacerbate SLE pathogenesis in a broad array of model systems (5-7). Furthermore, T cells isolated from SLE patients have intrinsic alterations in lipid composition, especially in specialized microdomains of the plasma membrane (also known as lipid rafts) that contain the $\mathrm{T}$ cell antigen receptor (TCR), and alterations in associated signaling molecules $(8,9)$. The combined weight of these observations argues that lipid metabolism influences self-tolerance and autoimmune pathogenesis; however, it is not known how defects in lipid metabolic programs direct the fate and function of immune cells in autoimmunity. In this issue, McDonald and colleagues shed new light on the complex crosstalk between lipid metabolism and $\mathrm{T}$ cell dysfunction by demonstrating that resetting glycosphingolipid (GSL) homeostasis partially restores TCR signaling and normalizes function in $\mathrm{T}$ cells isolated from patients with lupus (10).

\section{Alterations in $\mathrm{T}$ cell responsiveness associated with SLE}

It has long been appreciated that $\mathrm{T}$ cells purified from individuals with SLE are dysfunctional, particularly in patients with active disease (reviewed in ref. 1). Perhaps the best-characterized changes in $\mathrm{T}$ cells from SLE patients are profound alterations in TCR signaling. Detailed studies indicate that $\mathrm{T}$ cells from SLE patients display heightened calcium flux in response to antigen receptor stimulation. The molecular mechanisms underlying the acquisition of this "exaggerated" signaling phenotype 

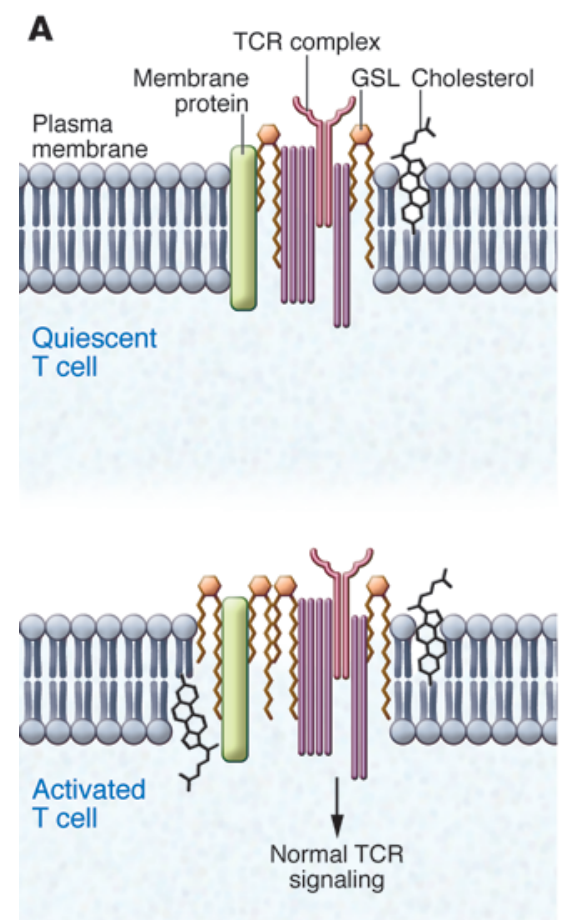

in SLE patients appears to be multifactorial. Biochemical characterization of the TCR signaling apparatus in T cells isolated from SLE patients indicates that changes in the assembly of the antigen receptor signal transduction machinery are mediated by the replacement of some signaling proteins $(8,11)$. The consequence of protein replacement is an alteration of the biochemical signaling pattern downstream of antigen receptor cross-linking and a subsequent perturbation of gene expression programs.

Multiple studies, including the current study by McDonald and colleagues, indicate that the alterations in TCR signaling are attributable, in part, to disruption of lipid homeostasis in the plasma membrane of SLE T cells (8-10). Specialized signal transduction localities within the plasma membrane, termed lipid rafts, rely on lipid composition to facilitate efficient TCR signaling. The lipid raft is specifically enriched with lipids, including cholesterol, sphingolipids, in particular sphingomyelin, and GSL species (Figure 1). The biochemical and biophysical properties of these lipids within the lipid raft are thought to facilitate the aggregation (or exclusion) of signal transduction machinery, and disruption of these microdomains can substantially alter TCR signaling (12). Interestingly, the characterization of $\mathrm{T}$ cells purified from SLE patients revealed alterations in GSLs and
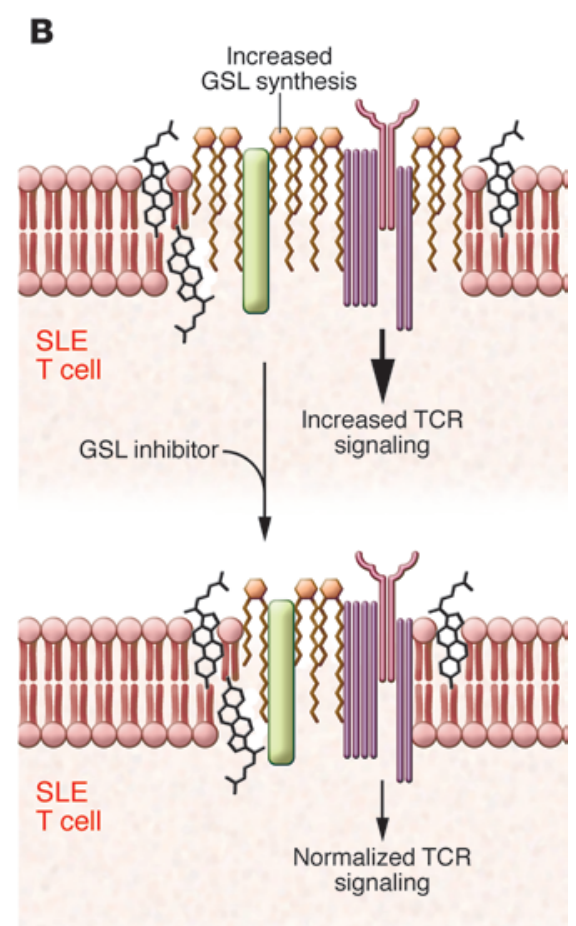

\section{Figure 1}

Resetting GSL homeostasis restores SLE $T$ cell dysfunction. (A) Lipid rafts are cholesterol- and GSL-rich microdomains in the plasma membrane that play important roles in regulating TCR signaling. In healthy individuals, the amount of cholesterol and GSLs is tightly regulated in quiescence. Activation increases both GSL and cholesterol levels in $T$ cells, which then return to quiescent levels following removal of activation signals. (B) SLE T cells have altered GSL and cholesterol homeostasis in lipid rafts that results in abnormal TCR signaling. Pharmacologic inhibition of GSL synthesis in SLE T cells restores GSL homeostasis, normalizes TCR signaling, and attenuates lupus $T$ cell dysfunction, indicating crosstalk between lipid metabolism and $\mathrm{T}$ cell function.

cholesterol within the plasma membrane, correlating with perturbations in lipid raft function $(8,9)$. These studies imply that lipid raft dysfunction may be a root cause of the exaggerated TCR signaling that is commonly observed in lymphocytes from SLE patients. In support of this assertion, disruption of lipid rafts or inhibition of cholesterol or GSL biosynthesis appears to be able to normalize TCR signaling and attenuate excessive cytokine production from autoimmune lymphocytes $(8,9,13)$. However, identification of the molecular mechanism(s) that drive lipid metabolic dysfunction in $\mathrm{T}$ cells from patients with rheumatic diseases has remained elusive.

\section{GSL changes: at the heart of autoimmune $T$ cell dysfunction?}

The first clue to identification of the players in SLE-associated $\mathrm{T}$ cell lipid dysfunction came from a detailed assessment of GSL composition. GSLs are a structurally diverse class of glycerolipids that are defined by the type of carbohydrate moiety bound to the lipid backbone (14). Using HPLC, McDonald and colleagues found that $\mathrm{T}$ cells from autoimmune patients have heightened GSL levels, which is consistent with previous findings $(8,9)$; however, HPLC analysis revealed that the composition of the GSLs was distinct from that found in activated $\mathrm{T}$ cells from healthy individuals. Further- more, the SLE-associated GSL pattern remained in place even when SLE T cells were cultured under "resting" conditions, suggesting that the unique GSL composition in autoimmune $\mathrm{T}$ cells is not a consequence of activation per se. Perhaps more provocatively, serum collected from SLE patients was able to increase GSL levels in the plasma membrane of $\mathrm{T}$ cells cultured from healthy individuals. These data clearly suggest that the lipid metabolic program of SLE T cells is a consequence of signals emanating from the host environment and imply that a generalized disruption of host lipid homeostasis underlies SLE-associated T cell dysfunction.

Based on their identification of a differential GSL expression pattern in SLE $\mathrm{T}$ cells, McDonald and colleagues examined whether the SLE-associated alterations were a function of altered GSL synthesis or turnover. Under normal conditions, cellular GSL levels are achieved through the combined effects of de novo synthesis, turnover, and recycling (15). The extent to which $\mathrm{T}$ cells in normal or disease states preferentially rely on one or more of these pathways remains largely unknown. Using a combination of fluorescent lipid tracers, organelle labeling probes, and pharmacologic inhibitors of organelle trafficking, McDonald et al. concluded that SLE T cells have both heightened GSL biosynthesis and increased 
trafficking to and from the plasma membrane, resulting in an aberrant accumulation of GSLs. Perhaps more importantly, pharmacologic inhibition of GSL biosynthesis in vitro with the clinically approved competitive inhibitor of glucosylceramide synthase NB-DNJ (16), a drug used to ameliorate lysosomal storage diseases, normalized GSL levels to those of healthy individuals and partially restored signaling defects in SLE T cells. Moreover, correction of GSL homeostasis in vitro ameliorated multiple facets of dysfunction and diminished the ability of these cells to drive autoantibody production from cocultured B cells.

\section{Liver $X$ receptors: regulators of lipid homeostasis and self-tolerance?}

So how do lupus T cells acquire this abnormal GSL metabolic phenotype? The observation that serum from SLE patients could induce lipid dysfunction in otherwise healthy $\mathrm{T}$ cells provided a clue, suggesting that a signal emanating from the host environment likely drives the metabolic program. McDonald and colleagues ruled out inflammatory signals in SLE-associated alterations of GSL biology and observed that the addition of oxidized lipoproteins (LDL) induced disease-associated GSL patterns in healthy $\mathrm{T}$ cells. Internalization of oxidized LDL is known to affect metabolism and inflammation through the actions of the lipid-regulated transcription factors liver X receptors (LXR $\alpha$ and LXR $\beta$ ). LXRs are members of the nuclear receptor superfamily that have an ever-expanding role in the transcriptional regulation of cellular lipid homeostasis (17). The LXRs are best known for their ability to transactivate genes involved in cellular cholesterol efflux (18); however, newer data indicate that LXRs also reduce lipoprotein import via the action of the E3 ligase IDOL (19) and induce phospholipid remodeling in membranes by the acetyl transferase LPCAT3 (20). Interestingly, LXRs are also able to repress inflammatory gene expression (e.g., IL6 and INOS), providing crosstalk between cellular metabolic status and inflammatory signaling (18).

Genetic ablation of both Lxra and Lxrb in mice results in a lupus-like disease, whereas pharmacologic activation of LXRs can attenuate lympho-proliferative disease and ameliorate autoantibody-mediated glomerulonephritis in spontaneous models of murine lupus (21). The molecular mechanisms underlying LXR-dependent mediation of self-tolerance are cell type depen- dent and multifactorial. Work from our lab and others suggests that LXR signaling facilitates the clearance of apoptotic bodies while suppressing inflammatory gene expression $(21,22)$. Likewise, LXRs can suppress proliferative capacity and differentiation of T lymphocytes $(23,24)$, likely through their ability to regulate cellular cholesterol. Thus, LXRs appear to be critical for coordination between self-tolerance and lipid metabolism.

McDonald and colleagues further asked whether LXRs could mediate the GSL dysregulation observed in human SLE T cells. Examination of LXR target genes indicated that $L X R B$ and genes involved in cellular lipid transport, such as the Niemann-Pick (NPC) proteins NPC1 and NPC2, were upregulated in isolated SLE T cells. Pharmacologic activation of LXRs transiently increased GSL expression in both healthy and SLE T cells; however, canonical LXR target genes, including $A B C A 1$ and $A B C G 1$, were not upregulated in SLE T cells, indicating an unusual pattern of LXR activity. Moreover, McDonald and colleagues noted that genes involved in cholesterol homeostasis, such as the sterol regulatory element-binding protein 2 (SREBP2) were also upregulated in SLE T cells. SREBPs are considered to be the master transcriptional regulators of lipid homeostasis through their ability to transactivate numerous genes involved in cholesterol biosynthesis and transport, including those encoding the NPC proteins $(25,26)$. GSL levels in lipid rafts are intimately tied to cholesterol homeostasis (15), and previous studies from Jury and colleagues have shown that inhibition of cholesterol synthesis can ameliorate many of the signaling abnormalities and dysfunction of SLE T cells (13). Thus, the question remains whether LXR signaling truly drives SLE-associated perturbations in GSL homeostasis, or whether LXR is activated to compensate for the dysregulation of other aspects of cholesterol metabolism in autoimmune $\mathrm{T}$ cells. Thus, it will be of interest in future experiments to dissect the apparent crosstalk between the SREBP and LXR pathways in $\mathrm{T}$ cells and the combined influence of these pathways on lipid raft function. It will also be intriguing to further investigate the potential of targeting GSL homeostasis in individuals with SLE as a therapeutic approach for attenuating autoimmune pathology. In conclusion, the provocative studies by McDonald and colleagues support the growing notion that metabolic reprogramming could provide a therapeutic avenue for ameliorating complex rheumatic diseases.

\section{Acknowledgments}

The authors were supported by grants from the NIH (AI093768, to S.J. Bensinger) and the Sontag Foundation (to S.J. Bensinger).

Address correspondence to: Steven J. Bensinger, UCLA, 36-120 CHS, Box 951735, Los Angeles, California 90095-1435, USA. Phone: 310.825.9885; Fax: 310.267.6267; E-mail: sbensinger@mednet.ucla.edu.

1. Tsokos GC. Systemic lupus erythematosus. N Engl JMed. 2011;365(22):2110-2121.

2. Roman MJ, et al. Prevalence and correlates of accelerated atherosclerosis in systemic lupus erythematosus. N Engl J Med. 2003;349(25):2399-2406.

3 . Asanuma Y, et al. Premature coronary-artery atherosclerosis in systemic lupus erythematosus. N Engl J Med. 2003;349(25):2407-2415.

4. Hahn BH, McMahon M. Atherosclerosis and systemic lupus erythematosus: the role of altered lipids and of autoantibodies. Lupus. 2008;17(5):368-370.

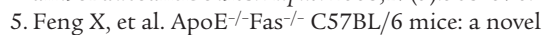
murine model simultaneously exhibits lupus nephritis, atherosclerosis, and osteopenia. J Lipid Res. 2007;48(4):794-805.

6. Gautier EL, et al. Enhanced immune system activation and arterial inflammation accelerates atherosclerosis in lupus-prone mice. Arterioscler Thromb Vasc Biol. 2007;27(7):1625-1631

7. Ma Z, Choudhury A, Kang SA, Monestier M, Cohen PL, Eisenberg RA. Accelerated atherosclerosis in ApoE deficient lupus mouse models. Clin Immunol. 2008;127(2):168-175.

8. Krishnan S, et al. Alterations in lipid raft composition and dynamics contribute to abnormal $\mathrm{T}$ cell responses in systemic lupus erythematosus. J Immunol. 2004;172(12):7821-7831.

9. Jury EC, Kabouridis PS, Flores-Borja F, Mageed RA, Isenberg DA. Altered lipid raft-associated signaling and ganglioside expression in $\mathrm{T}$ lymphocytes from patients with systemic lupus erythematosus. J Clin Invest. 2004;113(8):1176-1187.

10. McDonald G, et al. Normalizing glycosphingolipids restores function in $\mathrm{CD} 4^{+} \mathrm{T}$ cells from lupus patients. J Clin Invest. 2014;124(2):712-724.

11. Krishnan $S$, et al. Differential expression and molecular associations of Syk in systemic lupus erythematosus T cells. J Immunol. 2008; 181(11):8145-8152.

12. Jury EC, Flores-Borja F, Kabouridis PS. Lipid rafts in T cell signalling and disease. Semin Cell Dev Biol. 2007;18(5):608-615

13. Jury EC, Isenberg DA, Mauri C, Ehrenstein MR. Atorvastatin restores Lck expression and lipid raft-associated signaling in $\mathrm{T}$ cells from patients with systemic lupus erythematosus. J Immunol. 2006;177(10):7416-7422.

14. Lahiri S, Futerman AH. The metabolism and function of sphingolipids and glycosphingolipids. Cell Mol Life Sci. 2007;64(17):2270-2284.

15. Degroote S, Wolthoorn J, van Meer G. The cell biology of glycosphingolipids. Semin Cell Dev Biol. 2004;15(4):375-387.

16. Butters TD, Dwek RA, Platt FM. Imino sugar inhibitors for treating the lysosomal glycosphingolipidoses. Glycobiology. 2005;15(10):43R-52R.

17. Calkin AC, Tontonoz P. Transcriptional integration of metabolism by the nuclear sterol-activated receptors LXR and FXR. Nat Rev Mol Cell Biol. 
2012;13(4):213-224.

18. Bensinger SJ, Tontonoz P. Integration of metabolism and inflammation by lipid-activated nuclear receptors. Nature. 2008;454(7203):470-477.

19. Zelcer N, Hong C, Boyadjian R, Tontonoz P. LXR regulates cholesterol uptake through Idol-dependent ubiquitination of the LDL receptor. Science. 2009;325(5936):100-104.

20. Rong X, et al. LXRs regulate ER stress and inflammation through dynamic modulation of membrane phospholipid composition. Cell Metab.
2013;18(5):685-697.

21. A-Gonzalez N, et al. Apoptotic cells promote their own clearance and immune tolerance through activation of the nuclear receptor LXR. Immunity. 2009;31(2):245-258.

22. Hong C, et al. Coordinate regulation of neutrophil homeostasis by liver $\mathrm{X}$ receptors in mice. J Clin Invest. 2012;122(1):337-347.

23. Bensinger SJ, et al. LXR signaling couples sterol metabolism to proliferation in the acquired immune response. Cell. 2008;134(1):97-111.
24. Solt LA, Kamenecka TM, Burris TP. LXR-mediated inhibition of $\mathrm{CD}^{+} \mathrm{T}$ helper cells. PLoS One. 2012;7(9):e46615.

25. Gévry N, Schoonjans K, Guay F, Murphy BD. Cholesterol supply and SREBPs modulate transcription of the Niemann-Pick C-1 gene in steroidogenic tissues. J Lipid Res. 2008;49(5):1024-1033.

26. Horton JD, Goldstein JL, Brown MS. SREBPs: activators of the complete program of cholesterol and fatty acid synthesis in the liver. J Clin Invest. 2002;109(9):1125-1131.

\title{
Paradoxical insights into whole body metabolic adaptations following SGLT2 inhibition
}

\author{
William T. Cefalu \\ Pennington Biomedical Research Center, Baton Rouge, Louisiana, USA.
}

\begin{abstract}
It is well known that glycemic control over time reduces microvascular and macrovascular complications in human subjects with type 2 diabetes. In addition, preclinical models of type 2 diabetes have demonstrated that long-term hyperglycemia exacerbates insulin resistance and reduces $\beta$ cell function; therefore, therapies that reduce blood glucose levels are of great interest in not only controlling complications, but for restoring known defects in the pathogenesis of type 2 diabetes. Pharmacological inhibition of the sodiumglucose cotransporter 2 (SGLT2) reduces plasma glucose by limiting glucose absorption in the kidney and increasing glucose excretion in the urine. In this issue of the JCI, Merovci and colleagues and Ferrannini and colleagues independently report a paradoxical increase in endogenous glucose production in patients with type 2 diabetes following SGLT2 inhibition, despite an overall decrease in fasting plasma glucose. Together, these studies provide a unique insight into the effects of SGLT2 inhibition on whole body metabolism.
\end{abstract}

\section{Pharmacological control of hyperglycemia}

The natural history of type 2 diabetes (T2D) is characterized by a progressive decline in insulin secretion, development of insulin resistance, and increased hepatic glucose production. Based on these observations, it is generally accepted that after an initial period of lifestyle management and monotherapy (usually metformin), a combination of therapies designed to address T2D-associated pathophysiology will be required to effectively manage hyperglycemia over time (1). Currently, clinicians can chose from a large number of agents (e.g.,

Conflict of interest: William T. Cefalu has served as a consultant for Intarcia Therapeutics Inc. and, on behalf of his institution, has consulted for Shire, Novo Nordisk, and Sanofi-Aventis. He has served as principal investigator on research studies with funding awarded to his institution from AstraZeneca, Bristol-Myers Squibb, Sanofi-Aventis, Johnson \& Johnson, MannKind Corporation, GlaxoSmithKline, and Lexicon Pharmaceuticals Inc.

Citation for this article: J Clin Invest. 2014; 124(2):485-487. doi:10.1172/JCI74297. sulfonylureas, thiazolidinediones, incretin therapies, and exogenous insulin) that each have a unique mechanism of action, which makes each an appropriate add-on to the metformin background therapy (1). Although all of these agents are considered to provide efficacy on glycemic control, many of them produce adverse side effects that ultimately dictate their use. Thus, there is clearly a need for pharmacotherapy that can improve glycemia yet have a beneficial effect on unmet clinical needs, including weight gain and hypoglycemia. In this regard, there has been great interest in the sodium-glucose cotransporter 2 (SGLT2), because pharmacologic inhibition of this glucose cotransporter offers an attractive approach to modulate carbohydrate metabolism (2). Specifically, prior studies on SGLT2 inhibitors revealed that these agents lower the renal threshold for glucose and reduce glucose absorption in the kidney, resulting in increased urinary glucose excretion, decreased plasma glucose and glycated hemoglobin (A1c) lev- els, mild osmotic diuresis, and a favorable effect on weight (2-5). In essence, SGLT2 inhibition and associated net reduction in renal glucose reabsorption have provided a new insulin-independent approach for treatment of T2D.

To date, there are a number of SGLT2 inhibitors available currently or in development. Despite substantial clinical data on the effects of these inhibitors on glycemic control and other clinical parameters, there is a paucity of data in humans that comprehensively evaluates the whole body metabolic adaptation to pharmacologic inhibition of the renal SGLT2 cotransporter. In this issue of the JCI, results are presented from two elegantly conducted metabolic studies in individuals with T2D that evaluated the SGLT2 inhibitors, dapagliflozin and empagliflozin $(6,7)$. Both studies were well designed, used sophisticated metabolic techniques, evaluated subjects with T2D, involved both acute and longer-term evaluation (two- and four-week observations), and evaluated whole body insulin sensitivity and endogenous hepatic glucose production in response to SGLT2 inhibition. Although it appears that the primary aims and study approaches differed, it is interesting to note that both trials confirmed a surprising finding: SGLT2 inhibition elicits a paradoxical rise in endogenous glucose production (EGP), despite lowering fasting glucose.

\section{Testing the glucotoxicity hypothesis} in patients with T2D

In the study by Merovci et al. (6), the primary goal was to evaluate the impact of hyperglycemia and its reduction by SGLT2 